\title{
Calibration of LOFAR data on the cloud
}

\author{
Jose Sabater $^{\mathrm{a}, \mathrm{b}}$, Susana Sánchez Expósito ${ }^{\mathrm{b}}$, Philip Best ${ }^{\mathrm{a}}$, Julián Garrido ${ }^{\mathrm{b}}$, Lourdes Verdes-Montenegro ${ }^{\mathrm{b}}$, Daniele Lezzi $^{\mathrm{c}}$ \\ ${ }^{a}$ Institute for Astronomy (IfA), University of Edinburgh, Royal Observatory, Blackford Hill, EH9 3HJ Edinburgh, U.K. \\ ${ }^{b}$ Instituto de Astrofisica de Andalucía, CSIC, Apdo. 3004, 18080, Granada, Spain \\ ${ }^{c}$ Barcelona Supercomputing Center (BSC), Carrer de Jordi Girona, 29-31, 08034 Barcelona, Spain
}

\begin{abstract}
New scientific instruments are starting to generate an unprecedented amount of data. The Low Frequency Array (LOFAR), one of the Square Kilometre Array (SKA) pathfinders, is already producing data on a petabyte scale. The calibration of these data presents a huge challenge for final users: a) extensive storage and computing resources are required; b) the installation and maintenance of the software required for the processing is not trivial; and c) the requirements of calibration pipelines, which are experimental and under development, are quickly evolving. After encountering some limitations in classical infrastructures like dedicated clusters, we investigated the viability of cloud infrastructures as a solution.

We found that the installation and operation of LOFAR data calibration pipelines is not only possible, but can also be efficient in cloud infrastructures. The main advantages were: (1) the ease of software installation and maintenance, and the availability of standard APIs and tools, widely used in the industry; this reduces the requirement for significant manual intervention, which can have a highly negative impact in some infrastructures; (2) the flexibility to adapt the infrastructure to the needs of the problem, especially as those demands change over time; (3) the on-demand consumption of (shared) resources. We found that a critical factor (also in other infrastructures) is the availability of scratch storage areas of an appropriate size. We found no significant impediments associated with the speed of data transfer, the use of virtualization, the use of external block storage, or the memory available (provided a minimum threshold is reached).

Finally, we considered the cost-effectiveness of a commercial cloud like Amazon Web Services. While a cloud solution is more expensive than the operation of a large, fully-utilised cluster completely dedicated to LOFAR data reduction, we found that its costs are competitive if the number of datasets to be analysed is not high, or if the costs of maintaining a system capable of calibrating LOFAR data become high. Coupled with the advantages discussed above, this suggests that a cloud infrastructure may be favourable for many users.
\end{abstract}

\section{Introduction}

In the 21 st century, scientific research is being shaped by the exponential increase of the amount of data generated by new scientific instruments. Capture, curation, analysis, and sharing of these huge data volumes is a challenge that has triggered a new scientific paradigm: data-intensive science ('The Fourth Paradigm'; Tony Hey and Tolle, 2009). The astronomy community is preparing for the forthcoming Square Kilometre Array (SKA; Ekers, 2012), an instrument that once built, will be the largest scientific infrastructure on Earth and will achieve data rates on an exabyte scale. Currently some SKA pathfinders like the Low Frequency Array (LOFAR; van Haarlem et al., 2013) are already producing data on a petabyte scale. These scientific data together with those from the so-called 'Internet of Things', define the Big Data challenge.

To face this challenge, both powerful computing and highcapacity storage resources are required, as well as procedures capable of extracting relevant information from the data and

Email addresses: jsm@roe.ac.uk (Jose Sabater), sse@iaa.es (Susana Sánchez Expósito), pnb@roe.ac.uk (Philip Best), jgarrido@iaa.es (Julián Garrido), lourdes@iaa.es (Lourdes Verdes-Montenegro), daniele.lezzi@bsc.es (Daniele Lezzi) sharing it while ensuring reproducibility. Algorithms like MapReduce (e.g. Lämmel, 2008) have been key to process the unstructured data distributed on the Internet. However, they are not suitable for some scientific use cases, so scientists need to build procedures that are able to both process complex data and efficiently exploit the computing resources. A prime example of this is computational genomics, where algorithms like BLAST (Altschul et al. 1990) have taken advantage of supercomputing resources to empower genome sequence searches. The scientific community also investigates new computing infrastructures. The Large Hadron Collider (LHC) project designed a Grid tiered model, the Worldwide LHC Computing Grid (WLCG), that led to the creation of an European Grid Infrastructure open to other scientific communities. On the other hand, cloud computing is arising as a more flexible model than Grid computing or supercomputing, offering a virtual environment that is able to adapt to different use cases.

The LOFAR telescope is characterised by a new design that utilises software solutions to implement functionalities, like data correlation or source targeting, that have traditionally been performed by hardware cards or mechanical devices. The pipelines for reducing LOFAR data are high-computational-demand software, and indeed LOFAR capabilities are limited by the avail- 
able computing power instead of by the available observing time as in other existing telescopes. Therefore, in order to speed up the data processing, it is necessary to research both algorithms and computing resources.

We stress that the study that we present here was motivated by our real needs as final users (the users that receive the data from the observatory and attempt to exploit it scientifically) to calibrate LOFAR data (see also Dodson et al. (2016) for their similarly-motivated investigation into options for reducing and analysing spectral line data from the Very Large Array radio telescope). After the LOFAR data were successfully observed and delivered to us, it was clear that typical calibration strategies would not suffice for several reasons: a) the size of the data, with at least a couple of TB per observation, was large enough to require dedicated storage; $b$ ) the installation and update of the required specialized software was not trivial; c) the calibration process was experimental and the development of the new calibration strategies required frequent changes to the pipeline; and, d) the computational requirements of the pipeline were high enough to demand some type of parallelization. The solution widely adopted in the LOFAR community for the analysis of these data is the use of dedicated local clusters. After exploring the use of these, and also GRID infrastructures, we investigated whether a cloud infrastructure would be a suitable candidate technology for these analyses (Berriman et al., 2012). We tested different cloud infrastructures, and found that a cloud infrastructure that fulfils a given set of requirements may offer a solution for the calibration of new radio-interferometry data.

This paper presents the results of the tests that we performed on different computing resources, in order to evaluate which of them best fulfils the requirements from a new strategy for calibrating LOFAR data. In Section 2 we describe the LOFAR telescope and the challenge of calibrating its data. In Section 3 we outline the infrastructures tested, and we present the results of the preliminary tests performed in dedicated clusters and Grid infrastructures in Section 4 The description of the tests performed for the cloud infrastructures, and the results obtained, are presented in Section 5, including a comparison of costs relative to dedicated clusters. We review the suitability of the infrastructures tested in Section 6 and, finally, we present the summary, conclusions and future work in Section 7 .

\section{The Low Frequency Array, LOFAR}

The Low Frequency Array or LOFAR (van Haarlem et al., 2013, http://www.lofar.org/) is a new generation radio interferometer that covers low frequencies from 10 to $240 \mathrm{MHz}$. It is an interferometric array of 50 'stations' of relatively simple, low-cost dipole antennas, in which each antenna station has no moving parts, but instead operates as a phased array driven in software by powerful station-level computing. The core of the array is located in the north of the Netherlands and there are several remote stations across Europe. Due to its new design and technologies, it is considered to be one of the pathfinders of the Square Kilometre Array (Ekers, 2012). The output data from each LOFAR station is streamed (at up to $3 \mathrm{~Gb} / \mathrm{sec}$ per station) to a Central Processing facility based in Groningen, which handles real-time data operations such as the correlation of the data streams. The data output from the correlator is then streamed to an offline Central Processing facility, on which applicationdependent offline processes are run on the data. For standard survey imaging these may involve flagging of corrupted data (e.g. due to radio-frequency interference) followed by some averaging of the data in time and frequency to reduce data volume. There is local storage on the central processor to retain datasets for a short period of time whilst this processing occurs. The data are then transported to the Long Term Archive (LTA), where they are made available to users. The LTA is based on a Grid infrastructure (Holties et al., 2012).

Many of the SKA pathfinders are conducting large sky surveys (Norris et al. 2013) and LOFAR has already presented the first results from a shallow Northern-sky survey, the Multifrequency Snapshot Sky Survey (MSSS; Heald et al., 2015), a first step towards the production of deeper imaging surveys. These deeper surveys are being performed by the LOFAR Surveys Key Science Projects, which aims to explore the low frequency radio sky with an unprecedented depth (Röttgering et al. 2011). A component of this is the LOFAR Two-metre Sky Survey (Shimwell et al., 2016b) which is a wide area survey of the northern sky, reaching a typical r.m.s. within each 8-hr observation of $\sim 100 \mu \mathrm{Jy} /$ beam at $150 \mathrm{MHz}$, substantially deeper than any previous large-area radio survey (van Weeren et al., 2016a, Williams et al., 2016, Shimwell et al., 2016a; Hardcastle et al. 2016). Another component of the survey is to observe some selected fields, in which the highest-quality multi-frequency data exist, in two deeper tiers of observations. The work presented here was motivated by the need of calibration and analysis of one of the deepest fields: the ELAIS-N1 field (Right Ascension 16:08:44 and Declination $+56: 26: 30$ ). With about 200 hours of observations so far and more than $60 \mathrm{~TB}$ of pre-processed data in the LTA, the calibration and analysis of these data presents a formidable challenge.

\subsection{Description of the data}

In this paper, we focus on the generation of deep widefield radio images of the sky at low frequencies, ignoring all of LOFAR's other observation modes and techniques (e.g. Breitling et al. 2015). For our tests, we use an ELAIS-N1 field dataset, observed using LOFAR's High-Band Antennas (HBA; these cover the 110 to $240 \mathrm{MHz}$ frequency range). This observation is broadly typical of any LOFAR Surveys dataset: our results are thus generic for the calibration and imaging of all LOFAR HBA interferometric data.

Typical LOFAR imaging observation runs consist of: a) a 5-10 minute observation of a primary flux calibrator; b) several hours ( 5 to 8 hours, depending on the position of the target field and the data) on the main target, and either some flanking fields or a secondary target, using the multi-beam capacities of LOFAR; c) a final 5-10 minute run on a primary flux calibrator. The resulting LOFAR UV data is stored in CASA Measurements Set format (Kemball and Wieringa 2000, van Diepen, 2015). The field-of-view of the LOFAR HBA is of order 6 degrees (full width at half maximum; dependent on the observing 
frequency), with a beam resolution of $\sim 5$ arcseconds. The frequency coverage for the ELAIS-N1 data ranges from 115 $\mathrm{MHz}$ to $175 \mathrm{MHz}$ in 371 separate sub-bands. Each sub-band was originally composed of 64 spectral channels, and the initial scan-time was set to 1 second. During pre-processing, four of the edge-most channels were removed (due to poorer sensitivity) and the remaining data were averaged down to 15 channels per sub-band and a scan time of 2 seconds, reducing the size of the dataset by a factor of 8 . The typical size of a full preprocessed observation is of the order of 3 to $4 \mathrm{~TB}$.

The size of the datasets involved makes necessary a separation of the data based on different parameters. The calibration process usually takes advantage of correlations between the data to reduce the degrees of freedom of the problem and thus the bias of the fitting process (while not over-fitting; see van Weeren et al. 2016b). Hence, the parameters that are less correlated are selected to split the data:

Time: The UV data are usually correlated in adjacent time slots but there is little correlation in data taken far apart in time. This is particularly the case when data are split in different observing runs from different days. In this case, the final data model (description of the sources of the field) obtained in one observation can be used as an input model for another observation. Although the LOFAR data used in our tests is split in long chunks of 5 to 8 hours of data, the use of shorter time chunks is currently being tested.

Sky field: Different fields on the sky observed simultaneously (using multi-beam capabilities) contain different sources, unless they overlap. Usually their calibration solutions are substantially different (especially at low frequencies) and, in this sense, there is little advantage in processing the fields simultaneously. Therefore, the data are generally split into different sky fields that are processed separately.

Frequency: There are correlations between data at different frequencies that are affected by the drift of the reference clock signal of the observing stations (e.g. van Weeren et al. 2016b) and the effect of the plasma in the ionosphere. However, the structure of the data makes it easy to separate them in frequency ranges or sub-bands. The user can then decide how many of these sub-bands are required for a calibration in order to maximize the performance of the fitting process while keeping the size of the data manageable.

Fig. 1 shows a schema of the partition of data using the sky field, time and frequency parameters.

\subsection{Challenges posed by the data calibration}

LOFAR is currently completely operational and generating high quality data, whose correlation and pre-processing is performed routinely by the observatory. However, the calibration of the data by the final user still presents many challenges.

\subsubsection{Specialized software}

The calibration of the data requires dedicated LOFAR software. CASA (McMullin et al., 2007) can be used for some general tasks but some specialized tasks are only implemented by the LOFAR software compilation. These are mainly tasks that require the complex beam model of LOFAR, or that are optimized for the use on LOFAR data. The installation of the LOFAR software was especially difficult in old systems. It is relevant to take into account that the local computing resources available to the final user may be limited to these old systems. Although the complexity of the installation process has vastly improved due to the efforts of the LOFAR developers and packagers, the final user is required to maintain the software up-todate, in order to avoid critical bugs that are corrected in new releases. With several releases each year (e.g. 12 major releases in 2015 and 2016 plus several individual bug fixes), it may not be an easy task to keep the system up to date for a final user.

\subsubsection{High requirements on computing and storage resources}

A large amount of both storage and computing resources are needed for the calibration of these data. The final data set for a single observation run can amount up to several TB and a simple calibration run could take several CPU-years. However, the pipeline design allows the processing of different chunks of data (see Sect. 2.1) as independent jobs, which individually do not require a high amount of memory or processors. This suggests that a High Throughput Computing (HTC) system would fit better than a High Performance Computing (HPC) one. It is therefore advantageous to use powerful distributed computing infrastructures (DCIs) that may or may not be readily available to the final user.

\subsubsection{Varying hardware requirements}

One of the scientific challenges that LOFAR needs to overcome, and that will be even more demanding for SKA1 Aperture Arrays, relates to the necessity for 'direction-dependent calibration', which greatly increases the required computation. This arises both from the varying beam that the LOFAR stations produce, and the effects of the ionosphere, which are especially strong at low frequencies (Intema et al., 2009). In this latter regard, LOFAR poses one of the most difficult cases, with both a wide field of view and very long baselines, meaning that not only do different stations observe through a different patch of ionosphere, but within the imaged field-of-view of each station the ionospheric conditions are different towards different sources. The experimental pipeline developed by van Weeren et al. (2016b) aims to correct for these direction-dependent ionospheric effects. The pipeline has hardware requirements that have changed as it is developed. For example, new releases of the pipeline tend to require less memory and allow tasks to be run in parallel. On the other hand, the increased efficiency has permitted the processing of a higher volume of aggregated data in a single computing node, and the overall requirements are again increased. An alternative method of applying direction dependent calibration is also under investigation (Tasse et al. in prep.), and this has yet another different set of hardware 


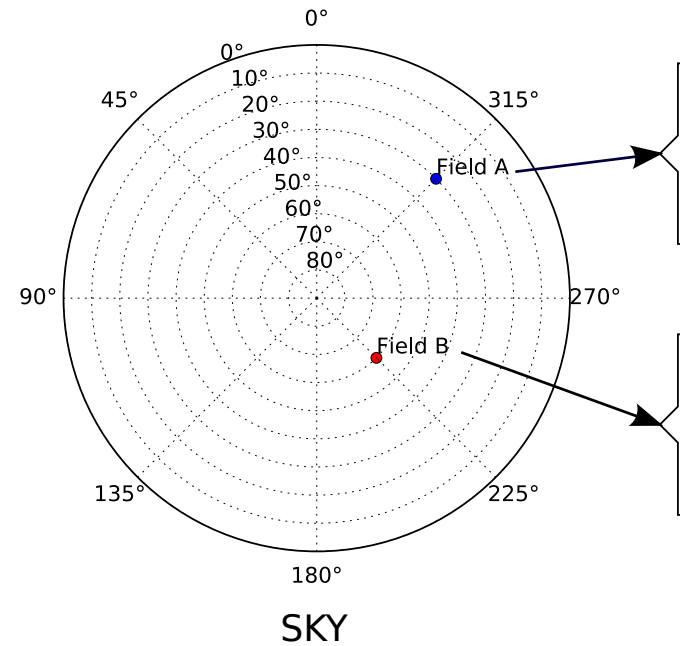

SKY
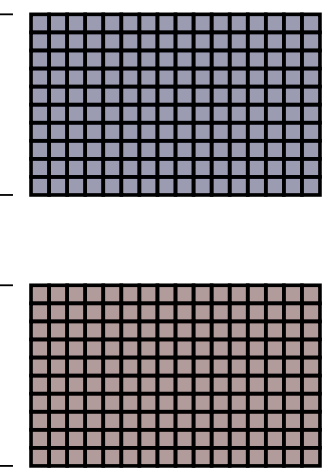

1

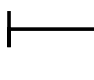

$+2$
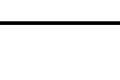

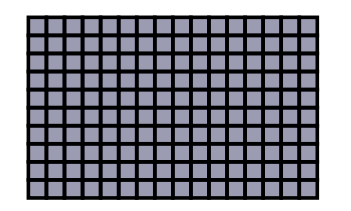

Figure 1: Partition of data in data chunks for parallel processing. They can be separated in different sky fields, time blocks and frequency blocks.

needs. The key general point here is that during the development process of new analysis techniques, although the global efficiency increases, the hardware requirements can often vary significantly. Flexibility is therefore a major advantage for the final user.

\section{Computational infrastructures investigated}

In this section we describe three different kinds of infrastructures in which we have performed our tests.

\subsection{Dedicated cluster}

The most widely adopted solution for the calibration and analysis of LOFAR data is the use of a dedicated cluster. We installed the LOFAR software and tested the pipeline described in Sect. 4 locally on the cluster at the Instituto de Astrofísica de Andalucía - Consejo Superior de Investicaciones Científicas (IAA-CSIC) and in the Stacpolly cluster at the Institute for Astronomy of the University of Edinburgh. We also tested the pipeline in the LOFAR-UK Computing Facility in the University of Hertfordshire. They are typical institutional supported clusters that use a PBS queue system to manage the jobs. The IAA-CSIC cluster and the LOFAR-UK Computing Facility use a shared file-system that is mounted in all the computer nodes while Stacpolly stores the data in a dedicated node.

\subsection{Grid infrastructure}

A Grid infrastructure was also considered for our tests. A computing Grid gathers the storage and computing resources provided by different sites or nodes, that can be seen as clusters connected through a Wide Area Network (WAN) running a common middleware that allows them to work as a single infrastructure. The access and the usage of the Grid resources are managed by means of Virtual Organisations (VOs). Users are grouped in VOs, and Grid nodes, depending on their institutional policy, support one or more VOs. Grid infrastructures provide a distributed parallel execution environment which is ideal to process this kind of data.

\subsection{Cloud infrastructure}

Finally, we considered a cloud solution which can offer the flexibility required by the target problem.

A cloud infrastructure provides shared computing, networking, data storage, or other services, on-demand in a flexible way. Most infrastructures offer the processing resources as computing instances (mainly virtual machines based on a configurable template or "image") that can be quickly provisioned or released. They can also offer object storage, in which the data are viewed as objects in a hierarchy, or block storage, in which data are treated as blocks of bytes like in classical disks and file-systems.

The tests were performed on three different cloud systems: a) an academic private multi-site cloud system, the European Grid Infrastructure (EGI) Federated Cloud, that brings together computing and storage resources from different national and intergovernmental European providers; b) an academic private one-site cloud managed by the Rutherford Appleton Laboratory (RAL), provided by the Science \& Technology Facilities Council (STFC) in the United Kingdom; and, c) a commercial multi-purpose public multi-site cloud, the Amazon Web Services (AWS) infrastructure.

The EGI Federated Cloud is a private cloud infrastructure open to any research community. It has been developed in the framework of EGI.eu and currently federates about 20 sites whose computing and storage resources are owned by different academic European institutions. In this infrastructure, computing and storage resources are provided through the standard Open Cloud Computing Interface (OCCI) and Cloud Data Management Interface (CDMI; ISO/IEC 17826:2016) and users access them with their X.509 certificates as member of a specific Virtual Organisation (VO). Once the user gets their proxy 
certificate, they can use it to gain access to the resources either through a Ruby or Java OCCI client or through high level tools built upon OCCI connectors that allow the user to manage the virtual resources. One example is COMPSs (Lordan et al. 2014), a programming model that, apart from being able to act as a cloud broker, optimizes the use of the computing resources through the exploitation of the inherent parallelism of the scientific applications.

Both the EGI Federated Cloud and the LOFAR LTA share a common Authentication and Authorization (A\&A) method based on a Public Key Infrastructure that uses the X.509 standard. This framework is defined by the European Policy Management Authority for Grid Authentication in e-Science Certification Authorities federation (EUGridPMA), and the Interoperable Global Trust Federation (IGTF). After obtaining their personal certificate, the users need to install the voms-client tool and to properly configure it. Users get a valid certificate proxy through this tool and can issue OCCI commands to the EGI Federated Cloud or request the transfer of data from the LOFAR LTA.

The Rutherford Appleton Laboratory Cloud offers another private cloud infrastructure open to the British scientific community. It is based on OpenNebula and provides mainly computing resources. The access to the infrastructure is provided by a custom dashboard and their installation of the OpenNebula Cloud operations centre called Sunstone. It is also possible to leverage the OpenNebula Application Programming Interface (API) endpoint using XML-RPC. Recently, a Ceph object store has been commissioned and integrated. Ceph offers an AWS-compatible API which simplifies the interaction with the system.

The third cloud system investigated was Amazon Web Services which is a suite of cloud-computing services provided by Amazon Inc. The resources are distributed in several regions (13 at the time of writing) around the world. We used the biggest one that is located in North Virginia (or "us-east1") to maximize the number of resources available. Among the services provided, we used Amazon Elastic Compute Cloud (EC2), Amazon Simple Storage Service (S3) and Amazon Route 53. EC2 is the Infrastructure as a Service part, providing the computing and block storage resources (Elastic Block Storage or EBS). S3 is the object storage service and Route 53 provides managed Domain Name System services. We interacted with the services using the web-based AWS console and the AWS API through the Python Boto library or their command line interface software.

The SKA Organization published in 2015 a joint call for proposals with Amazon (Astrocompute) to investigate and develop radio-astronomy tools and processes in the AWS infrastructure. Most of the AWS tests shown here were carried within the context of one Astrocompute project proposed by the authors and consumed the credits (equivalent to money that can be spent on AWS resources) provided for the project. The usage of resources provided by AWS is billed monthly and the amount depends on the type of resource and its usage.

\section{Preliminary tests in dedicated clusters and the Grid}

For most of the tests presented in this paper we used a simplified pipeline in which the data from just one sub-band are calibrated, corrected and imaged. These are the basic steps of a typical self-calibration loop and contain the fundamental steps used in calibration pipelines of radio-interferometric data. The calibration and correction are run using the LOFAR Black Board Selfcal (BBS) system (Pandey et al., 2009) and the imaging is done with the AWimager (Tasse et al., 2013). BBS runs in one core at the time of making the tests. The data are loaded in memory in chunks whose size is manually selected using an input parameter. AWimager has multi-core and automatic memory managing capabilities. Although the advanced pipelines presented in van Weeren et al. (2016b) are also being adapted to the cloud by the authors, the tests using the simplified pipeline cover the basic performance issues that can be found in the advanced ones.

In order to check the general capability of the infrastructure to run the pipeline in parallel, we ran the pipeline on several instances in parallel as a test. The calibration of a full dataset would require us to run in parallel chunks of data obtained from the combination of several sub-bands. However, the test on those instances was enough to check how the infrastructure responded to the parallel run of the pipeline, while retaining the option to manually check possible errors and reduce the time of the individual tests. All of the infrastructures involved responded as expected to the parallelization and the calibration time using several instances took practically the same time as the calibration of a single band in one instance (as long as there were enough resources available).

In this section we describe our tests in two different kind of HTC systems: clusters and Grids. The results of the test performed in the cloud infrastructures will be present in detail in the next section.

\subsection{Dedicated cluster}

One of the main issues that drove us to consider other solutions was the difficulty to install and keep updated the required software on the clusters. The installation on the IAA-CSIC cluster was done first and took several weeks. The long time required for the installation was mainly caused by the relatively old version of the operating system in comparison with the libraries required by the LOFAR software. Almost all of the dependencies had to be manually compiled, installed and configured as a local user. The installation on Stacpolly took less time due to the experience acquired in the other cluster, the relatively new operating system, and the release by third parties of scripts that helped with the installation of the dependencies. However, the software became quickly outdated and some new dependencies appeared. It had to be manually recompiled which was time consuming.

Although the cluster solution works at present, it may not scale well in the future when the load of computing and storage requirements increase. Furthermore, at the time of writing, the requirements of the newest advanced pipelines are higher than the capacity of the nodes of the the IAA and the Stacpolly 
clusters; this highlights a potential difficulty of the inflexibility of dedicated clusters during the development phase of new analytic techniques.

\subsection{Grid infrastructure}

The problems with the installation and update of the software were similar to those shown for the clusters with an additional difficulty arising from the use of different operating systems in each site. Additionally, the installation and deployment of the user application may require the intervention of the system administrators of each provider of the Grid infrastructure where the pipeline would run. This may be impractical whilst the calibration pipeline is under active development and frequently changing. However, this kind of solution could be considered in the future to implement a stable calibration pipeline. This scenario would be more favourable if the deployment of the software is eased by, for example, the use of containers (see Docker; Boettiger, 2015).

Once the pipelines are stable, it will be possible to run them using the computational resources provided by the same Grid site in which the data are stored. This would reduce significantly the overhead due to the transfer of data. This approach is currently being explored by the LOFAR Surveys Key Science Project team on the SURFsara site.

\section{Calibration of radio-astronomy data in the cloud}

We performed several tests to assess the suitability and performance of the cloud platforms used to solve our problem. We checked the following points:

Installation and update of the software: We tested how easy it was to install and manage the required software in the infrastructure. We also considered how easy it was to keep the software up to date and the level of human intervention and support required.

Suitability of the platform: We investigated the suitability of the platform to run the pipeline and to be adapted to it. We first checked if the platform was capable of providing the resources required by the pipeline. We also checked the ability to adapt it easily to new requirements of the pipeline.

Data transfer: We transferred a high volume of data from the LOFAR LTA to some of the infrastructures. We determined the speed of the transfer and the level of human intervention required.

Processing performance: We studied whether it was possible to run the pipeline efficiently in parallel in the cloud distributed environment. We tested several parameters like the running time, memory consumed, load of the processors, etc.

Cost: Finally, we investigated the cost of the commercial cloud that we used, AWS, and compared it to the cost of other infrastructures.

\subsection{Software installation and update}

The installation and update of the software in the infrastructure was one of the most time consuming processes. As mentioned in the previous section, we had previously tried the manual compilation and installation of the LOFAR dependencies by trial and error in a relatively old Red Hat operating system used by one of the clusters. This experience was crucial to consider a cloud infrastructure as a good alternative. A cloud infrastructure allows to select one base operating system that is convenient for the installation of the LOFAR software. The software is installed once in an image that is used as the base for all the computing instances.

For the installation of the LOFAR software in the EGI infrastructure we used the same approach that we followed for the cluster in Edinburgh. We run an automatic installation script provided by Astro Wise. The script downloaded fixed versions of the dependencies from the Astro Wise server that are known to work. It required some manual tweaking and the manual installation of some additional dependencies to adapt it to our needs. Although the approach is powerful, as it is independent of the Linux flavour and provides compiled software that is optimized for the target operating system, there are some caveats: a) the version of the dependencies is fixed; b) there is no automatic way to upgrade the system of dependencies; c) the lack of a custom required dependency that is not implemented in the system is usually detected at run-time; and, d) it could take a considerable time to compile all the dependencies. Additionally, the maintenance of a generic script like this can be cumbersome and, apparently, it is no longer maintained.

Overall, we followed the recommended method to install user applications on the EGI Federated Cloud. We created, by means of an OCCI client, a VM using a Virtual Appliance published in the EGI Federated Cloud Applications Database formerly known as the Application Database Cloud Market (AppDB; https://appdb.egi.eu/). Later, the software was installed accessing the VM through SSH public/private key pair. Once the user application (the LOFAR and CASA software in our case) was installed, we created a VM image from a snapshot of the VM (this process could only be done by site administrators at time of performing these tests). After that, we uploaded the image to the AppDB. Finally, we requested the inclusion of the image in the VO image list so it could be deployed on the Federated Cloud sites supporting the VO.

For the RAL and AWS cloud infrastructures we used installation and provisioning recipes created in Ansible. Ansible is an automation tool used for application deployment, configuration management and orchestration. It can be interfaced using "recipes" written in YAML which make them easy to read and write by human beings. The recipes are divided by functionality to allow a granular installation of software and an easy upgrade ${ }^{1}$ Ansible permits us to use the same recipes in different systems like the RAL federated cloud based on OpenNebula and the AWS infrastructure. 2

${ }^{1}$ The recipes are released as playbook roles in https://github.com/nudomarinero/Astrocompute-ELAIS-N1

${ }^{2}$ We did not use Ansible in EGI because the installation process that we 
We used the Long Term Releases of Ubuntu (12.04 and 14.04) as our base operating system. At first we created our own Ubuntu Personal Package Archives (PPA) with some custom changes on the radio astronomy packages created by Gijs Moolenar. Later, the relevant changes were implemented in the main "radio-astro" PPA. This PPA was used as the base for the LOFAR software dependencies. However, the main LOFAR package is still compiled separately to allow a quicker response to bug patches and software upgrades. Please note that the radio-astro PPA is being superseded by the new Kern Suite

The previously mentioned effort to package the dependencies of LOFAR (mainly casacore casarest and pyrap or pythoncasacore) in an Ubuntu PPA allows the integration of the software in the standard packaging system with all its advantages (automatic processing of dependencies, easy installation and removal of files, etc.) - though also with some of its disadvantages, like the possible lack of compiler optimization.

Regarding the creation of the base image or template for the instances, for the RAL cloud the software was installed in an instance running a base Ubuntu system. A snapshot of the instance was created and later published as a base image. This step required some support from the RAL. For AWS we used Packer ${ }^{3}$ to create an Amazon Machine Image (AMI) automatically. In this case no human intervention was required at all.

Table 1 shows a summary of the installation of software and the creation of the base image in different infrastructures.

It should be noted that the level of human intervention required can have an impact on the usability of the infrastructure. If the step requiring human intervention has to be repeated often then the time consumed by this can easily add up to a considerable amount, which makes it impractical. In our case the software has to be updated often and the level of human intervention had a considerable impact on the time required to implement our tests.

\subsection{Suitability of the infrastructures}

The calibration pipeline is under development and its requirements change. At the time of writing this paper, it needs several tens of GB of memory and a local scratch data area of at least $1 \mathrm{~TB}$ that is used to hold the intermediate data. It benefits from multiprocessing in most of the steps. We needed infrastructures that can adapt to those requirements.

A wide range of machine sizes helps in the development of a pipeline that optimises the usage of resources. A machine with a large number of CPU cores can speed up the execution of tasks that are adapted to multiprocessing but its resources can remain largely idle if a single core application needs to be run. From a user point of view it is sometimes very difficult or even impossible to modify or adapt the underlying software to optimize the usage of resources. In this context it makes sense to utilise a cloud infrastructure in which the sizes of the machines can be adapted to the optimization level of the software

\footnotetext{
followed on EGI required manual tweaking and we did it only once. However, we can not think of a technical reason to not to be able to use Ansible in EGI.

3"Packer is a tool for creating machine and container images for multiple platforms from a single source configuration"; https://www.packer.io/
}

available. The capacities of relevant instance types in AWS, in the EGI Federated Cloud used for the tests (in our case we used CESNET), and in the RAL cloud, are summarized in Table 2, It is important to note that the CPUs of the AWS instances are actually virtual CPUs that made use of the Intel Hyper-threading technology (Marr et al. 2002), that means that their performance is roughly half of the performance of a physical CPU. This correction factor will be taken into account for the comparisons.

The amount of memory required for the pipeline, particularly for the imaging process, could sum to dozens of GB. In the two clusters, one of the technical constraints was the limited and fixed amount of memory available on each node. In some cases, the computing nodes did not have enough memory to perform the imaging step at an adequate resolution. Most of the cloud infrastructures provided a wider range of choices for the node sizes including instances with large memory. We needed the possibility to launch VMs with this high amount of memory.

One particularity of AWS is the availability of non-reserved instances with variable prices that follow the market demand, called spot instances. Usually their prices are several times lower (they range from $\approx 10$ times lower to being even 10 times more expensive) than those of standard reserved instances and are ideal to run processes that do not require real time responsiveness like in our case. A maximum bidding price is set when an instance is requested and the instance runs until the market price rises over this limit. In this event the instance is automatically shut down. When using spot instances the pipeline must be adapted to allow it to be resumed in the event of an instance shut-down. In our case we used spot instances whenever it was possible. It is important to note that the probability of a shut-down of the spot instance anti-correlates with the maximum bidding price; a relatively high price will allow the instance to run uninterrupted for a longer time. Hence, longer processing times usually require higher bidding prices to avoid interruptions. We will discuss the final price per instance that we empirically calculated in Section 5.5

There were several options for the storage, each with advantages and disadvantages:

Shared file-system: One option used in one of the clusters was the use of a shared file-system (GlusterFS). Several dedicated nodes provided access to the storage area which was visible from all the computing nodes. The I/O rate could be limited by the local network connection which could be an issue depending on the problem. However, the simplicity of use of these systems could compensate the possible loss of performance during I/O operations. This option is currently available in AWS (Amazon Elastic File System) but was not used for our tests.

NFS mount: Another simple option was the use of an NFS (Network File System; Sandberg, 1986) mount. This configuration was used in one of the EGI Federated Cloud sites. Although it worked eventually, this solution presented several disadvantages: a) the set up of the system 
Table 1: Software bundling and base image preparation in each infrastructure.

\begin{tabular}{lll}
\hline Infrastructure & installation of software & creation of base image \\
\hline \hline cluster Granada & manual installation & - \\
cluster Edinburgh & installation script & - \\
EGI Federated cloud & installation script & human intervention \\
RAL cloud & PPA+custom LOFAR package & Semi-automated with Ansible and human intervention \\
AWS cloud & PPA+custom LOFAR package & Automated with Packer and Ansible \\
\hline
\end{tabular}

Table 2: Type of instances used for the tests in each cloud infrastructure.

\begin{tabular}{|c|c|c|c|}
\hline instance type & $\begin{array}{l}\text { cores } \\
(\mathrm{N})\end{array}$ & $\begin{array}{l}\text { memory } \\
(\mathrm{GB})\end{array}$ & type of storage \\
\hline \multicolumn{4}{|c|}{ AWS } \\
\hline m4.large & $2^{*}$ & 8 & EBS (450 MB/s) \\
\hline m4.xlarge & $4^{*}$ & 16 & $\mathrm{EBS}(750 \mathrm{MB} / \mathrm{s})$ \\
\hline $\mathrm{m} 4.2 x$ large & $8^{*}$ & 32 & EBS (1000 MB/s) \\
\hline $\mathrm{m} 4.4 x$ large & $16^{*}$ & 64 & EBS (2000 MB/s) \\
\hline m4.10xlarge & $36^{*}$ & 160 & EBS (4000 MB/s) \\
\hline c4.xlarge & $4^{*}$ & 7.5 & EBS (750 MB/s) \\
\hline c4.2xlarge & $8^{*}$ & 15 & EBS (1000 MB/s) \\
\hline c4.4xlarge & $16^{*}$ & 30 & EBS $(2000 \mathrm{MB} / \mathrm{s})$ \\
\hline c4.8xlarge & $36^{*}$ & 60 & EBS (4000 MB/s) \\
\hline m3.large & $2^{*}$ & 7.5 & EBS and $1 \times 32$ GB int. \\
\hline m3.xlarge & $4^{*}$ & 15 & EBS and $2 \times 40$ GB int. \\
\hline $\mathrm{m} 3.2 x$ large & $8^{*}$ & 30 & EBS and $2 \times 80 \mathrm{~GB}$ int. \\
\hline c3.xlarge & $4^{*}$ & 7.5 & EBS and $2 \times 40 \mathrm{~GB}$ int. \\
\hline c3.2xlarge & $8^{*}$ & 15 & EBS and $2 \times 80$ GB int. \\
\hline c3.4xlarge & $16^{*}$ & 30 & EBS and $2 \times 160 \mathrm{~GB}$ int. \\
\hline c3.8xlarge & $36^{*}$ & 60 & EBS and $2 \times 320 \mathrm{~GB}$ int. \\
\hline r3.large & $2^{*}$ & 15.25 & EBS and $1 \times 32$ GB int. \\
\hline r3.xlarge & $4^{*}$ & 30.5 & EBS and $1 \times 80 \mathrm{~GB}$ int. \\
\hline \multicolumn{4}{|c|}{ EGI Federated Cloud (CESNET) } \\
\hline mem_medium & 2 & 8 & NFS \\
\hline extra_large & 8 & 8 & NFS \\
\hline mem_extra_large & 8 & 32 & NFS \\
\hline mammoth & 16 & 32 & NFS \\
\hline \multicolumn{4}{|c|}{ RAL Cloud } \\
\hline Lofar-Ubuntu-14-8c-32gb-2TB & 8 & 32 & Internal 2TB \\
\hline
\end{tabular}

* Virtual hyperthreaded cores. 
required support from the site administrators; b) its configuration and set up was not accessible by the API; and, c) it was a custom solution that could not be extended to other sites and required the development of site-specific steps on the testing pipeline.

Object storage: Object storage is used to store large data files and it is ideal to store the input data, intermediate data objects between the main calibration blocks in large pipelines, and final output data. Although this is very useful to store large volumes of data for extended periods, the files can not be handled as regular files in a file-system by the software.

Block storage: Block storage can be attached as data volumes to the VM instances. They can be used as scratch areas that are resilient to instance shut-downs. They can be used to locally store the intermediate data as they are viewed as normal disk by the operating system. Block storage is usually offered by cloud platforms as one of their services but this is not always the case. Although this type of storage is very convenient, the I/O rate could be limited depending on the configuration (see Sect. 5.4) and it is usually more expensive than object storage. Hence, a mixed storage system is usually used with block storage.

Internal storage: Internal storage is provided in some clouds with some types of instances and is equivalent to a typical scratch data area. This type of storage is ideal to locally store the intermediate data, and offers the best performance in terms of $\mathrm{I} / \mathrm{O}$ speed. However, such storage is usually ephemeral and, in general, the data is wiped once the instance is shut down. This can be a problem if the instance can be shut down before the full completion of the calibration pipeline, as in the case of spot instances in AWS.

One of the main problems that we did not foresee until testing the pipeline on different infrastructures was the effect of limited availability of scratch data areas (internal or block storage). The data required for a calibration run must be present in the instance in an standard file-system. Even after splitting the data in fields, observation runs and frequency bands, the size of these datasets ranges from several hundreds of GB to a few TB. Additionally, some space for the final data products and for intermediate-step data is also required. Hence, a VM instance requires an standard file-system of the order of TBs attached. The lack of internal or block storage, or some limits on the size of the block storage devices at the time of performing the tests, prevented us from finishing the implementation of the full calibration pipeline in all platforms except AWS.

One of the points that had a big impact on the usability of a platform was its maturity. More mature platforms usually present more options and a solid ecosystem of tools to leverage them. For example, the usage of the libraries and APIs of Open Stack or AWS was very easy. Although these points are not purely technical, they had an impact on the time that we spent setting up the tests and solving the problems that we encountered.

\subsection{Data transfer}

We checked the data transfer to two cloud infrastructures that offered object storage. We used S3 in AWS and the new Ceph cluster at the RAL.

The transfer of the data from the LOFAR LTA requires the use of common A\&A X.509 credentials as explained in Sect. 3.3 The software used for the transfer was the "SRM" suite and is based on the Storage Resource Manager protocol. One dataset (a single observation run ranging from 5 to 10 hours) was transferred at a time and the process was the following:

1. A proxy certificate for the Grid credentials with the longest valid time (one week) was created.

2. A dataset, composed of several data files (usually from 244 to 371 files), was staged in the LOFAR LTA servers using a XML-RPC petition. During this process the data was moved from tapes to a temporary disk storage area (the staging area) that can be directly accessed for a period of time.

3. The data was downloaded in parallel from one VM instance in the target infrastructure. As soon as a data file was downloaded it was uploaded to the object storage service provided by the infrastructure. For this process we wrote some simple Python scripts

The AWS S3 and Ceph cluster each provide a compatible API. The API can be accessed using the Python library Boto in the same way for both infrastructures. The only difference was a small set of configuration parameters. This compatibility made it very easy to write common software for the upload of data that could be used in both infrastructures.

We transferred 18 full datasets from the LOFAR LTA to the AWS S3 facilities in North Virginia (us-east-1 region). The volume of each individual dataset is about 3 to $4 \mathrm{~TB}$ with a total size of $\approx 64 \mathrm{~TB}$. An instance of type m4.10xlarge was launched and provisioned using Ansible. This type of instance offered the highest available bandwidth and a large number of processors to allow a parallel download. We attached an EBS volume as a temporary data area that was used to store the partial data files in the process between the start of the download and the end of the upload to the object storage service. After testing a number of parallel downloads between 18 and 36 (36 was the number of processors of the instance), we selected 24 parallel downloads as the optimal number that maximized the transfer speed whilst maintaining the stability of the instance. We think that the stability problems with high concurrencies originated from the saturation of the network connection which is also used to communicate to the EBS filesystem.

The transfer started mid-September 2015 and finished midDecember 2015 as shown in Fig. 2. There are several gaps in the transfer process and removing them the total transfer time could be reduced from $\approx 3$ months to $\approx 1.5$ months. However, most of these gaps were very difficult to avoid and probably present a realistic scenario to be found in semi-supervised data 


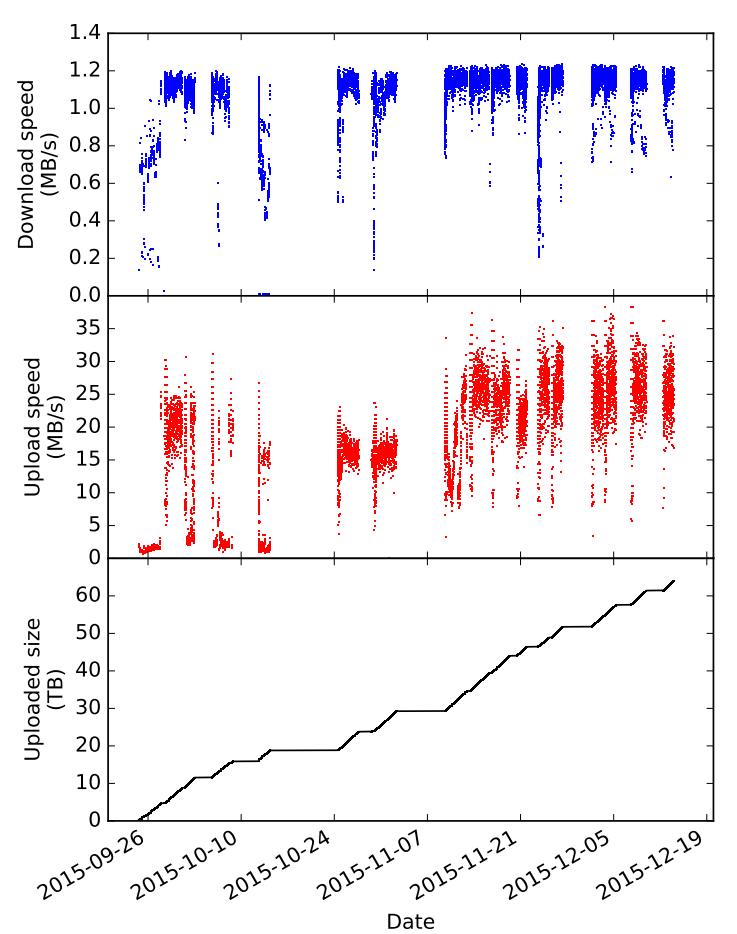

Figure 2: Data transfer to AWS S3. The upper panel shows the speed of transfer for each of the data files from the LOFAR LTA to the AWS EC2 instance in the us-east-1 region. The mid panel shows the speed of transfer of the data files from the AWS EC2 instance to AWS S3. The lower panel shows the cumulative size of the data uploaded to S3.

transfer processes carried out by final users like in this case. The transfer speed for each file was measured by dividing its transfer time by its size. The transfer speed from the LOFAR LTA to the AWS infrastructure peaks at about $1.2 \mathrm{MB} / \mathrm{s}$ for each data file. The mean upload speed to S3 (that uses the internal AWS network) ranges from less than 15 to more than $35 \mathrm{MB} / \mathrm{s}$ for each data file. This variance is probably affected by the number of parallel uploads and we estimate that the total upload speed at a given point is very similar.

The gaps in the data transfer had different origins. Apart from the testing nature of this experiment that made us to spend some time in tests that are not shown in Fig. 2, we had some issues with the staging of data and the manual renewal of the proxy certificate. The time required for the staging of data in the LOFAR LTA varied widely from time to time, probably due to the load on the service. On one occasion the data disappeared from the staging area. This was probably caused by the limited storage space of the staging area, an issue that is currently being solved by the LOFAR LTA. Hence, all the staging process had to be supervised to spot and solve possible problems. Additionally, the download from the LTA required the creation and usage of a proxy certificate. This process could only be done interactively and the maximum duration of the proxy certificate was one week. Therefore, the process required constant supervision.

We also uploaded one of the datasets to the Ceph cluster at the RAL. The process was similar to the upload to AWS. We used an internal VM instance that was provisioned with Ansible and an attached scratch data volume in the RAL cloud. Although we could not save the exact profiling data for this test, the transfer speed from the LOFAR LTA to the RAL infrastructure was several times (about 3 or 4 times) higher than the transfer to the AWS infrastructure. We assumed that this was mainly caused by a higher bandwidth of the connection between the two European sites.

Finally, we would like to mention a possible alternative method to transfer data: the physical transfer of storage units (disks, tapes, etc) between data centres. After we started our tests, AWS started offering a new service of data transfer called Snowball. A unit of $50 \mathrm{~TB}$ of solid disk storage with high bandwidth local network connectivity could be sent by courier to transfer the data between the data centre and AWS S3. At the time of writing this paper, the physical transfer of data could outperform the transfer via the regular Internet network.

\subsection{Processing performance}

We focused our performance tests on the EGI Federated Cloud Infrastructure and on the AWS infrastructure.

We ran the simplified test pipeline using one single ELAISN1 sub-band centred at $115.037537 \mathrm{MHz}$ with one frequency channel, 61 antennas (1891 baselines) and 2879 time slots in an 8 hours observing run (10 s scan time). The size of the data was $\approx 1 \mathrm{~GB}$. The pipeline was run in several instance types in AWS and the EGI Federated cloud (see Table 2). We measured the time spent in each phase of the calibration pipeline.

In Fig. 3 we show the time taken in the "solve", "correct" and "image" steps of the pipeline. The number of CPU cores is normalized to physical cores and the time is also normalized. That means that the number of cores and times are divided by 2 for AWS data to allow a better comparison of the trends with the number of cores. We plot the data for the AWS and EGI Federated clouds and, as a comparison, the Stacpolly and LOFARUK clusters. In Fig. 4 there are several panels showing the CPU and memory usage and the data $\mathrm{I} / \mathrm{O}$ for different calibration test runs. In this figure it is possible to see the CPU and memory consumption of each step as well as the cumulative I/O.

In the "solve" step (upper panel in Fig. 3) the data is calibrated to an input model using BBS. The program uses only one core and it is not parallelized. This can be seen as the flat relation with the number of cores and the similar times in the two cloud infrastructures. The running times for the Stacpolly and Hertfordshire clusters are higher, at least in part due to the use of less powerful processors. This step could be treated as an embarrassingly parallel problem and several bands can be run in parallel in a single node or instance to maximize the performance. However, if the number of "solve" processes running in parallel in a single computing node is lower than the number of cores (for example, if there are less bands to process than cores) some of then will be idle with a corresponding waste of resources. In this case, the use of cloud instances with sizes adapted to the number of bands to process is one advantage of cloud infrastructures. The "solve" step can be seen in the initial part of the panels in Fig. 4 with one or two flat peaks of memory 

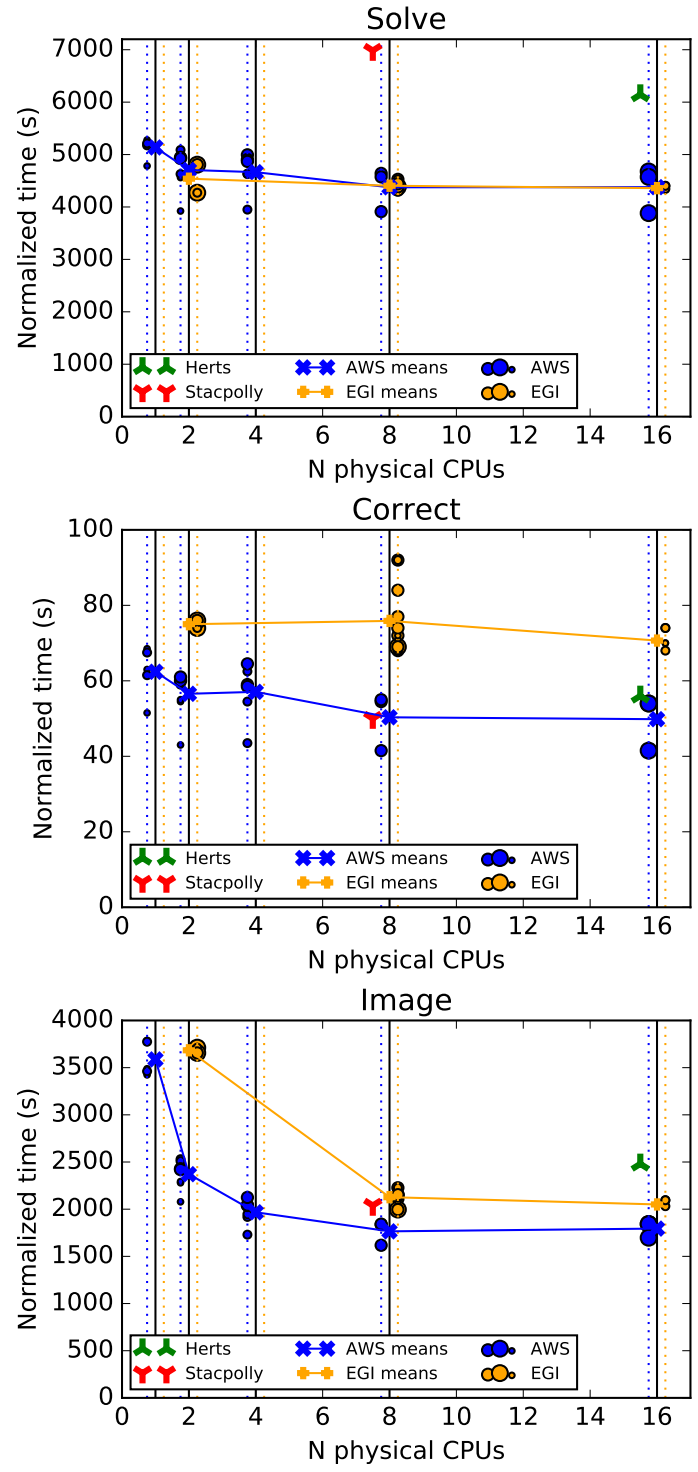

Figure 3: The time taken by the Solve, Correct and Image steps for our tests in different infrastructures. The CPU core numbers are normalized to physical CPUs (divided by a factor 2 in AWS). We show the individual tests in AWS (blue) and the EGI Federated Cloud (orange) cloud infrastructures as a cloud of points on either side of the main line that corresponds to the number of CPUs and the means in the centre. The size of the points is proportional to the memory of the instance used. As a comparison, we show the tests in the cluster Stacpolly in Edinburgh (red) and the LOFAR-UK Computing Facility in Hertfordshire (green). They are shown with a slight offset in $\mathrm{x}$ position for clarity. usage. In those cases the CPU core usage is approximately 100 per cent and the speed of the step is mainly determined by the CPU clock speed.

The "correct" step (middle panel in Fig. 3 involves applying the solutions computed in the "solve" step to the data and writing the corrected data back to the disk. It is computationally very simple and involves some data $\mathrm{I} / \mathrm{O}$. The times are very short in comparison with the "solve" step and the dispersion of the times is higher. In Fig. 4 the step is barely visible between the "correct" and "image" step given its short duration. The running times are very similar in the clusters and AWS and slightly higher in the EGI Federated Cloud, probably due to some overheads related to the use of an NFS file-system.

Finally, in the "image" step (lower panel in Fig. 3) the data is imaged using AWImager. Part of the imaging process is run in parallel making use of all the available processors. There is an improvement in the running time as the number of cores is increased. However, after a certain point there is no further significant improvement. Although the most computationally demanding part of the computing is parallelized, there is a part that runs in only one core. The time spent in this part cannot be improved using more cores and the time decreases asymptotically to the time spent on this part when we increase the number of cores (Amdahl, 2007). This step can be seen in the last part of the panels shown in Fig. 4. The parallel part uses all the CPU resources but the time spent in this part decreases as the number of cores increases while the single core part remains broadly constant.

With the "image" step it is also possible to see the combined effect of hyperthreading and the lack of complete parallelization in a process. In Fig. 5 we show the real time spent in the step and the number of physical cores used. For the same number of physical cores we always get better performance in the EGI Federated Cloud infrastructure that does not use hyperthreading. The overheads found in AWS with respect to EGI are $29 \pm 8$ per cent with 2 physical cores; $66 \pm 13$ per cent with 8 physical cores; and, $75 \pm 9$ per cent with 16 physical cores. When hyperthreading is used, doubling the number of cores has little effect on the speed of the parallelized part (double the processors at half the speed) but the single core part runs at a lower speed (about half the speed). This can be seen in Fig. 4 , where the parallelized part (seen as peaks in the CPU usage in the last part of the profile) tends to be shorter with a higher number of cores while the single core part (the flat part with values close to 100 per cent between the peaks) remains similar in length. We observe that hyperthreading is only equivalent if several processes can be run in parallel until they use all the processor resources, or if the software runs fully in parallel. The difference in CPU usage paterns between the "solve", "correct" and "image" steps could be matched in a cloud infrastructure by a correct selection of the instance type. Additionally, if the cloud provides persistent block storage (like EBS in AWS), the single-core steps can be run in single-core instances and the parallelized steps in many-core instances working on the same data areas as required by the pipeline. This flexibility is one of the advantages of a cloud infrastructure.

For our tests, the effect of the memory available on the in- 

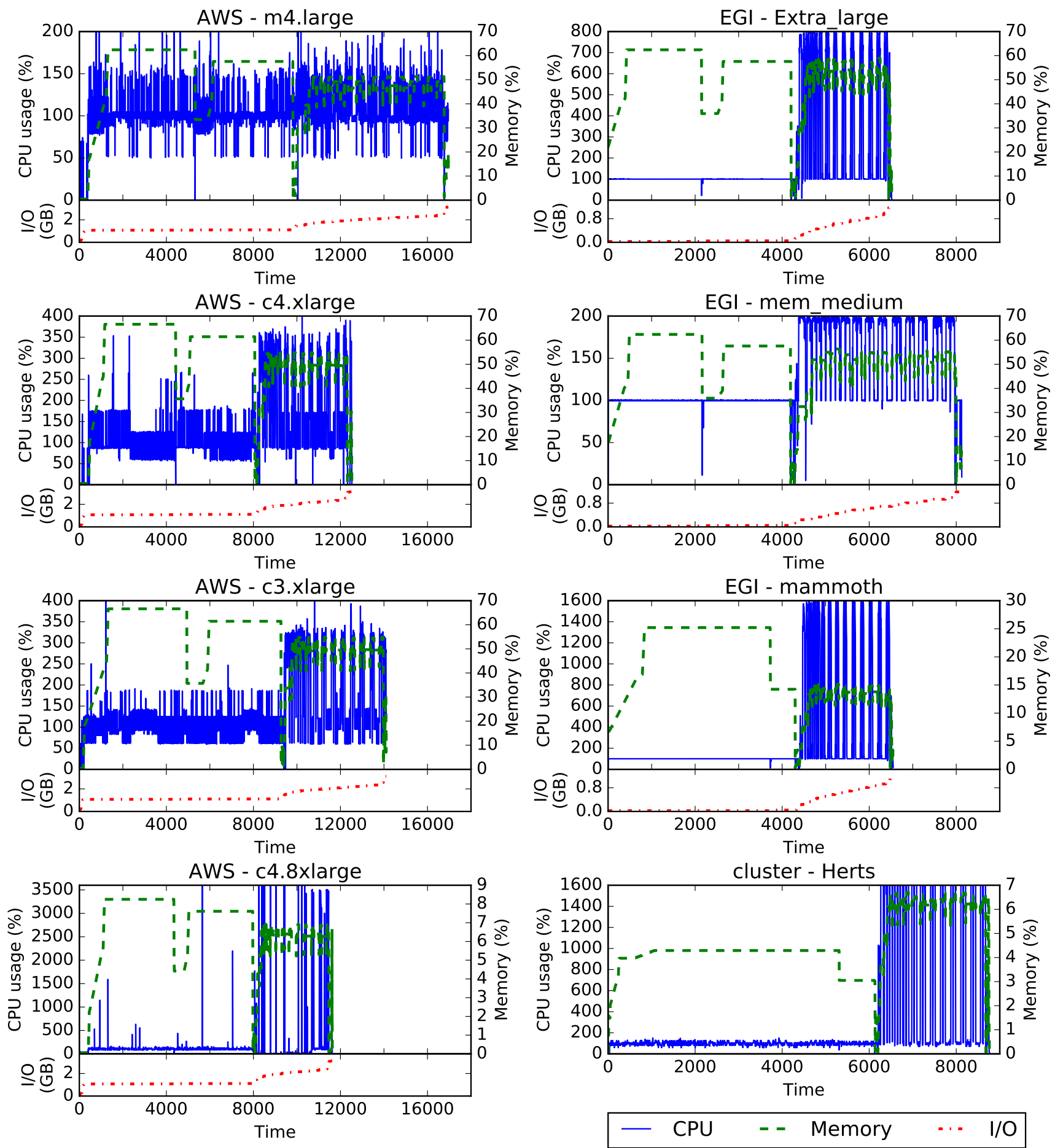

Figure 4: CPU and memory usage (blue solid and green dashed lines in the upper part of each panel) and input/output usage (red dash-dot line in lower part of each panel) for different instance types in the AWS and EGI Federated cloud infrastructures. The CPU and memory usage of the LOFAR-UK cluster in Hertfordshire is shown in the lower-right panel as a comparison. The AWS profiles include the initial data download step at the beginning (visible as a quick jump in the I/O usage). The "solve" and "correct" steps are at the beginning and consume approximately one core because they do not use multiprocessing. The "solve" step can be seen as the one or two flat peaks in memory usage and the "correct" is barely visible given it short duration. At the end, the "image" step is clearly visible with burst of parallel CPU usage and continuous I/O of data. 


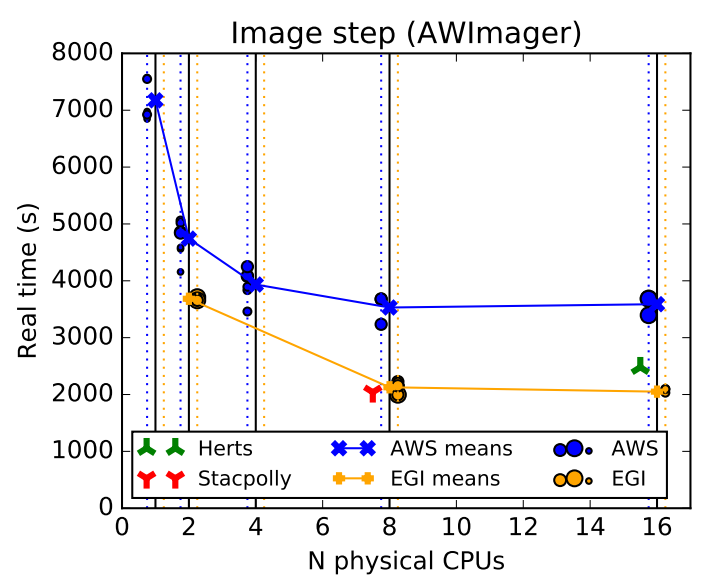

Figure 5: Time spent in the "image" step with respect to the number of physical cores of the instance or node. The symbols are as described in Fig. 3

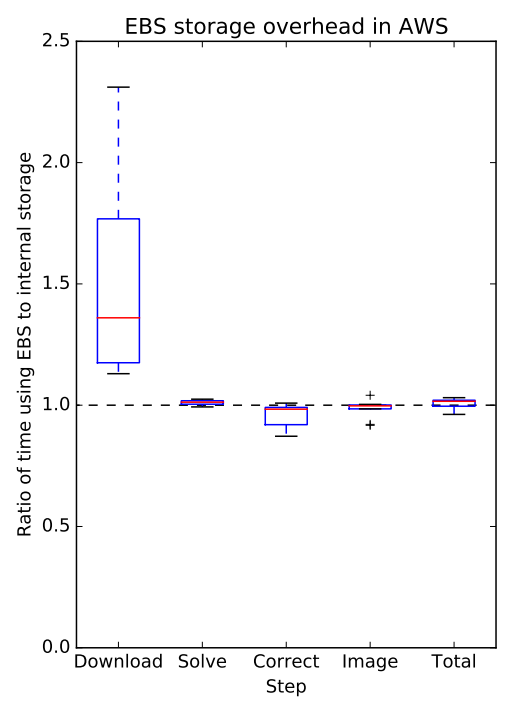

Figure 6: Relative time differences between EBS and internal storage volumes in the AWS infrastructure. The box and whiskers plots show the distribution of the fraction of time spent using an EBS volume with respect to using internal storage for each step. stance or node was not significant once a minimum threshold was reached. All the tests that run in instances with less than 8 GB of memory failed (bad_alloc errors), but after this threshold was reached the performance was very similar, as shown in Fig. 3. The size of the scattered dots indicates the memory of the instance and, once the number of processors is fixed, only has a marginal effect on the time spent in the step. The memory threshold depends strongly on the size of the dataset. Tests with datasets that were 5 times bigger required a minimum of 32 GB of memory. The memory requirements depend strongly on the particular software used, the pipeline run, the number of processes running in parallel and the size of the dataset.

We also measured in AWS the performance of different steps ("download", "solve", "correct" and "image") in instances with different types of storage. By comparing the same instances using either internal storage or EBS storage we could determine if there is a significant overhead related to the use of EBS (Dodson et al. 2016). The results are shown in Fig. 6. The "download" step is where the data were transferred from the object store to the internal storage and was only measured in AWS. Given the high bandwidth available, the time of the "download" step is mainly dominated by the $\mathrm{I} / \mathrm{O}$ performance. In this case we found a median overhead of $\approx 36$ per cent over using internal storage. In the other steps there was no overhead within the error. We could not find a significant overhead in the overall test pipeline that depends on the use of EBS (the total overhead is $0.5 \pm 2.4 \%$ )

One last point to consider in terms of performance is the effect of interruptions in the processing. With AWS spot instances the chance of a shut down of the instances increases with longer running times. Our simple tests were short but advanced pipelines take longer to run. In those cases we found it fundamental to be able to resume the running of the pipelines. Hence, it could be useful to design the pipeline software with well defined intermediate states from where it can resume the computing.

\subsection{Costs}

We made an estimation of the costs of processing real LOFAR data in the AWS infrastructure versus using a dedicated cluster. There are many factors that can affect the accuracy of our estimate. One of the main factors is the optimization of the pipeline and infrastructure usage in AWS which is under development. For example, we are currently experimenting with improvements that can reduce the costs by more than 40 per cent, but we provide the current numbers as a general guideline. We do not consider in this comparison the price of long term object storage in the infrastructure (e.g. S3) and focus on the computing prices.

In a commercial cloud infrastructure the computing resources are consumed as they are used and they are usually charged at a unit rate per unit of time. In a dedicated cluster the initial price of the machine should be considered in addition to the running cost (electricity, support, etc). If the running cost of the dedicated cluster is lower than the cloud price, the time in which investing in a dedicated cluster pays off is given by the 
equation:

$$
t p_{\text {cloud }}=c_{\text {cluster }}+t p_{\text {cluster }},
$$

where $t$ is the time, $p_{\text {cloud }}$ is the cost of the cloud per unit of time, $c_{\text {cluster }}$ is the initial investment on the cluster, and $p_{\text {cluster }}$ is the running cost of the cluster per unit of time.

For our estimation we used a real calibration run of a combination of 40 sub-bands of one of the ELAIS-N1 datasets. The calibration followed the pipeline described in van Weeren et al. (2016b) and was run using the software called factor It took 380 hours to complete (just over two weeks). The final prices on AWS were $\$ 177.75$ for the computing with an m4.4xlarge instance and $\$ 157.77$ for the EBS storage, adding up to a total of $\$ 335.52$, or $\$ 0.883$ per hour. The use of spot instances reduced the price from $\$ 0.958$ per hour to a mean of $\$ 0.468$ per hour, that is 49 per cent of the nominal price of the instance. There was one interruption during the calibration process produced by the shut-down of the instance due to a spike in market prices. The m4.4xlarge instance that we used in AWS has 16 vCPUs (8 physical cores of an Intel Xeon E5-2684 v4 at 2.3 $\mathrm{GHz}$ or E5-2676 v3 at $2.4 \mathrm{GHz}$ ), $64 \mathrm{~GB}$ of RAM memory and a 3 TB EBS solid state disk volume. We got a quote for a server with similar characteristics of $\approx \$ 9500$ (November 2016; exchange rate 1.25 USD to GBP). We considered in this case a running cost of $\$ 4000$ per year ( $\$ 1000$ electricity charges and $\$ 3000$ for the human support). Using eq. 1 we derived a time of 2.5 years for the costs of the two systems to be equivalent.

We can estimate the number of datasets that can be processed during this time. In our case, the time expended was 380 hours for the 40 sub-bands, although the on-going efforts to optimise the software and selection of instances is continuing to improve this. The calibration of the 18 ELAIS-N1 full datasets would require to run 162 times this quantity of data. Hence, the computed 2.5 years correspond to just over a third of the $162 \times 40$ sub-band runs.

The parameters used in our estimation can vary widely from case to case. For example, the cost of the support will be much lower if the computing resource is integrated into an existing cluster or if the electricity is already paid for the final user by third parties. We present in Table 3 different possible scenarios and their estimated break-even times and number of full datasets that can be calibrated in this time obtained from eq. 1 .

We consider 4 different hardware infrastructures: a) an 8core node with 64 GB or memory and 3 TB of solid state disks for the scratch area with a network connection of $10 \mathrm{Gbit} / \mathrm{s}$; b) the same option but with spinning disks; c) 16 cores with 128 GB of memory and 6 TB of solid state disks; and, d) 64 cores with $1 \mathrm{~TB}$ of memory and $12 \mathrm{~TB}$ of spinning disks. We also consider a range of operational scenarios which depend mainly on the context and support. The "data centre" scenario means that the user has access to a local data centre with specialised support in which to place the computing resource. This scenario provides a lower limit to the cost of the cluster with the electricity provided and a support cost of $\$ 400$ per year (based on a cost $\$ 50$ per year per core; E. Tittley private communication). In the "no electricity" scenario the support is estimated at $\$ 3000$ per year but the electricity is already provided by the centre. The "base" scenario is that considered above, where the user also has to pay the electricity consumed by the computing. In the "alone" scenario we raise the price of the support to $\$ 10000$ per year to account for the considerable amount of the time that the user or a third party must expend in maintaining the computing node or infrastructure. Finally, the "dedicated" scenario considers that one person $(\$ 50000$ per year) is fully dedicated to maintain the computing infrastructure. Although this scenario is not realistic, it marks an upper limit to the cost of the cluster.

As we can see in Table 3 a bigger (dedicated) computing resource could outperform the AWS cloud in terms of costs. However, the size of the problem to solve must be relatively big (a considerable number of datasets need to be calibrated) and the money for such a resource must be available. For smaller and cheaper computing resources, the AWS cloud could offer a cheaper solution when the cost of the support is relatively high or the number of datasets to calibrate is not very high.

There are, however, several further differences to consider between the cluster and the cloud approach. A cloud infrastructure like AWS allows the parallel processing of several datasets, which may be interesting with tight deadlines. The obsolescence of the hardware was not taken into account in the previous calculations. The cost of AWS tends to go down steadily with time while the performance of the hardware is improved simultaneously. Furthermore, these calculations assume the cluster is used at 100 per cent efficiency: for intermittent use there will be wasted downtime, whilst on a commercial cloud you pay nothing for this.

\section{Review of infrastructure suitability}

In this section we combine our experiences and test results from our investigations of different infrastructures, to present some general considerations that are relevant for the calibration and analysis of LOFAR (or other comparable) data in any computing platform. We then review the issues specifically relevant to clouds.

\subsection{General considerations for all infrastructures}

One of the main problems that can be found in any infrastructure occurs if there is a lack of support or documentation, or complexities in their usage. These factors have a great impact in the time consumed developing the calibration pipelines, to the extent of rendering some computing platforms practically unusable, particularly for a final user with limited time. The processes that were complex or unclear were especially time consuming requiring a lot of trial and error. This problem can be accentuated in bespoke solutions where the support of a wide community of users is not available. Good support, good and up to date documentation and the simplification of usage 4 , had a very positive impact on the developing time.

\footnotetext{
4"Simplicity is a great virtue but it requires hard work to achieve it and education to appreciate it. And to make matters worse: complexity sells better." (Dijkstra 1984)
} 
Table 3: Break-even times and the corresponding number of processed datasets estimated for different hardware infrastructures and operational scenarios.

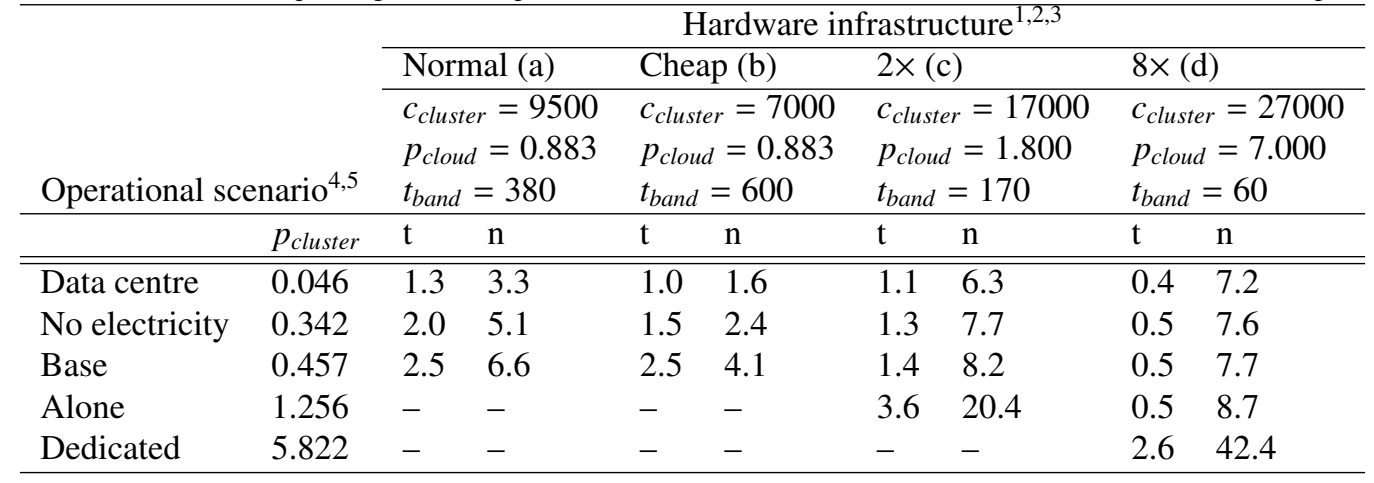

${ }^{1}$ A detailed description of the hardware infrastructure can be found in the main text.

2 The units of $c_{\text {cluster }}$ are $\$$; for $p_{\text {cloud }}$ are $\$$ per hour; and, for $t_{\text {band }}$ are hours. A full dataset is composed of 9 bands.

3 The columns are: (1) t, time in years to break-even the costs of AWS and the cluster; (2) n, number of full datasets that can be calibrated in this time. A "-" indicates that the costs of AWS are always lower for reasonable lifetimes (ie. $t>5$ yrs).

${ }^{4}$ A detailed description of the operational scenarios can be found in the main text.

5 The units of $p_{\text {cluster }}$ are $\$$ per hour.

The requirement of manual intervention for some tasks had a strong impact on the time spent. The difficulty of synchronizing agendas, the overload of support staff, the temporal unavailability of key contacts, can all have an added negative impact when human intervention is required. This effect can add up quickly if many of the steps involved require this intervention or if it is required by simple tasks. This problem is minimized with the automation of the infrastructures. The time spent in this regard in fully automated infrastructures, like AWS, was minimal.

Regarding infrastructures, some of the main concerns in terms of performance are related to: the efficiency and configuration of the processing power; the memory available; the speed of the data transfer; and, the efficiency of the data I/O.

In terms of processing speed, we found some effects that depended on the processor (processor speed, generation, etc.) but that were of second order. Multiprocessing was used in (parts of) tasks that were parallelized with the corresponding reduction of the processing time. The computational overhead due to virtualization in clouds was minimal (if the number of virtual CPU cores corresponded to the number of physical cores; we discuss the use of multiple virtual cores per physical core later). In general we found the cloud infrastructures tested to perform similarly or better than the clusters in terms of CPU speed. Many other factors had a much greater impact than this.

The memory was not a factor that limited processing performance, in general. The pipelines required a minimum amount of memory to run without failing. Once the instance or computing node provided this minimum amount, the availability of additional memory had little impact on the processing time of our test pipelines. The memory problems can be mitigated with new memory optimized software and a correct sizing of the resources.
The speed of the data transfer was initially thought to be a limiting factor but we did not find it to be a bottleneck on the overall process. The transfer times were lower than the processing times and orders of magnitude lower than the time spent developing the solutions in this early stage. During the data transfer step, the main overheads came from the requirement to manually supervise the transfer process (staging of data, creation and renewal of the proxy certificates, error checking, etc.) but not from the actual transfer of data.

The efficiency of the data $\mathrm{I} / \mathrm{O}$ can have a potential impact on the speed of the calibration depending on the efficiency of the underlying system. This is particularly the case for our tests and some of the pipelines under development that are I/O bound instead of CPU bound. We did not find a large data I/O overhead coming from the use of non-local storage in the clouds. Specially, the use of EBS volumes in AWS had only a limited impact, less than $\approx 36$ per cent in the download of data step and practically null in the overall calibration tests. However, we found the lack of proper size scratch storage areas to be a limiting factor in many of the infrastructures that we tested.

The availability and size of scratch storage areas turned out to be of great importance for the implementation of the pipelines. In many cases, we found that the pipelines required more scratch storage than the infrastructures were able to provide and this blocked the implementation. The Elastic Block Storage service of AWS proved to be very useful in this sense.

\subsection{Advantages and disadvantages of cloud infrastructures}

The cloud infrastructures excelled in the simplicity for the software installation and maintenance. Once the base operating system was ready, the installation of software and creation of instances with an exact copy of the software was straightforward. 
The availability of standard APIs and tools facilitated greatly the integration of the pipeline. Those APIs and tools (e.g. Ansible, Boto, or Ipython (Pérez and Granger, 2007)) are widely used in the industry by communities out of the scientific circles which allows a transverse transfer of knowledge. The APIs of AWS or Open Stack were well documented and supported which permitted a quick integration of the software developed.

The flexibility of the cloud infrastructures allowed to adapt the hardware to the requirements of the pipelines, which is fundamental in the early stage of testing. Apart from that, this flexibility allows to optimize the consumption of resources once the calibration pipeline is well defined. All the cloud infrastructures permitted the parallel execution of the pipelines with a number of parallel instances that was only limited by the size of the cloud infrastructure or the resources allocated to the user. In the case of AWS the limits were very high allowing the parallel development of complex pipelines and the experimentation with different infrastructure parameters (instance size, volume sizes and types, etc.).

We found an issue that can be present in any infrastructure that uses virtualization but particularly on the cloud. The combination of Hyperthreading (where the number of processors is duplicated but the apparent clock speed is halved) with software that is not fully parallelized had a negative impact on the processing speed in the pipelines tested. The apparent lower speed per processor combined with the fact that parts of some processes did not, or could not, use multi-processing, caused an overhead in the overall run time (see Amdahl, 2007). Whilst hyperthreading may present advantages for other users, we found in our tests in AWS that the overhead in time was as high as 75 per cent in some steps of the pipeline. The performance would be lower overall but the real extent of the impact should be assessed with additional tests.

The model offered by the clouds, where the resources are consumed on-demand, could be of advantage in shared infrastructures where resources are allocated and consumed by a wide range of scientific projects and fields that may have different technical requirements. For a final user it offers the advantage of just paying for the usage of resources without the commitment required by dedicated infrastructures.

The costs associated to a commercial cloud like AWS can be equivalent or lower than those of a dedicated cluster in some common cases. It is a cost effective way of processing data if the user needs to calibrate low to medium volumes of data, or if they need otherwise to use a shared system in which the admin costs of maintaining up-to-date LOFAR software become high.

\section{Summary and conclusions}

New scientific instruments are starting to generate an unprecedented amount of data. The capture, curation and analysis of these huge data volumes require the use of innovative strategies. The forthcoming Square Kilometre Array (SKA) will achieve data rates on a exabyte scale. LOFAR, one of the SKA pathfinders, is already producing data in a petabyte scale whose calibration present a formidable challenge. The several TB of data for each observation, a software whose installation and maintenance was not trivial, and a calibration pipeline that was quickly evolving and required intensive storage and computing resources, motivated us to investigate the use of different computing resources. After considering dedicated clusters and Grid infrastructures for the calibration of our LOFAR imaging data from the point of view of a final user, we focused mainly on cloud infrastructures. A cloud infrastructure can provide the flexibility and high throughput for the calibration of the big volumes of radio-astronomy data that we are handling.

Our initial tests on dedicated clusters were mainly limited by the complexity of the software installation and maintenance in these systems. An additional problem was the increase of computational resources required by the pipelines which rendered some of the clusters unable to run them. We also explored the use of Grid infrastructures but the need of manual intervention combined with the quick development of the pipelines at this early stage made them unsuitable for our needs. However, they are an infrastructure that should be evaluated once the pipelines are in a stable state. After that, we performed our tests in different cloud infrastructures: the EGI Federated Cloud, the RAL Cloud, and the commercial cloud Amazon Web Services.

In general we found that good support, documentation, and simplicity of usage were of great importance for the implementation of the pipelines: the requirement of manual intervention had a strong negative impact on the time spent in some infrastructures. On the technical side, processing speed was comparable in the different infrastructures for similar resources. The quantity of memory had little impact on the processing speed once a minimum amount of required memory was available. The speed of data transfer was not one of the main limiting factors as the transfer time was lower than the computing time. Finally, we could not find a strong data I/O overhead coming from the use of non-local storage in the clouds.

However, we identified a couple of unforeseen issues that had a negative impact on the implementation of pipelines. The combination of hyperthreading and tasks of the pipelines that are not, or cannot be, fully parallelized produced an empirical overhead in the running time of the pipelines. Additionally, the lack of scratch storage areas of an appropriate size could block the implementation of the pipelines in some systems.

Cloud infrastructures presented several highlights, most notably: a) the straightforward and simple installation and maintenance of the software; b) the availability of standard APIs and tools widely used in the industry; c) the flexibility to adapt the infrastructure to the needs of the problem; and, d) the ondemand consumption of shared resources.

We found that the run of data calibration pipelines is not just possible but efficient in cloud infrastructures. From the point of view of the final user it simplified many important steps and solved issues that blocked the implementation or running of the pipelines in other infrastructures. In terms of costs, a commercial cloud infrastructure like AWS is currently worthwhile in several common use cases, where the user lacks access to powerful storage and computing resources or specialised support, or where the calibration of small to medium sets of data is needed.

In the future we will present the detailed results and technical details of the calibration of the LOFAR ELAIS-N1 data on 
AWS. We are currently optimising and integrating in AWS the full calibration pipeline presented in van Weeren et al. (2016b We also plan to perform tests in other clouds and continue with the study of the integration in the EGI and RAL clouds. We will test the use of COMPs and, once the pipeline is stable, the integration of the full pipeline in a Grid infrastructure will be considered.

\section{Acknowledgements}

We acknowledge the useful comments of the anonymous referee. We would like to acknowledge the work of all the developers and packagers of the LOFAR software that constitute the core of the processing pipelines (including factor, prefactor, LSMTool, LoSoTo, and the Kern Suite), as well as the useful discussions with the participants in the LOFAR blank fields and direction dependent calibration teleconferences over the years. JS and PNB are grateful for financial support from STFC via grant ST/M001229/1. This work has been also supported by the projects 'AMIGA5: gas in and around galaxies. Scientific and technological preparation for the SKA' (AYA201452013-C2-1-R) and 'AMIGA6: gas in and around galaxies. Preparation for SKA science and contribution to the design of the SKA data flow' (AYA2015-65973-C3-1-R) both of which were co-funded by MICINN and FEDER funds and the Junta de Andalucía (Spain) grants P08-FQM-4205 and TIC-114. We would like to explicitly acknowledge Dr Jose Ruedas - chief of the computer centre and responsible of the computing and communications infrastructures at IAA-CSIC - and Rafael Parra system administrator of the IAA computing cluster - for their technical assistance. We acknowledge the joint SKA and AWS Astrocompute proposal call that was used to fund all the tests in the AWS infrastructure with the projects "Calibration of LOFAR ELAIS-N1 data in the Amazon cloud" and "Amazon Cloud Processing of LOFAR Tier-1 surveys: Opening up a new window on the Universe". This work made use of the University of Hertfordshire's high-performance computing facility and the LOFAR-UK computing facility, supported by STFC [grant number ST/P000096/1]. This work benefited from services and resources provided by the fedcloud.egi.eu Virtual Organization, supported by the national resource providers of the EGI Federation. We acknowledge the resources and support provided by the STFC RAL Cloud infrastructure. LOFAR, the Low Frequency Array designed and constructed by ASTRON, has facilities in several countries, that are owned by various parties (each with their own funding sources), and that are collectively operated by the International LOFAR Telescope (ILT) foundation under a joint scientific policy.

\section{References}

\footnotetext{
${ }^{5}$ The current efforts can be found in http://www.lofarcloud.uk
} 